Electrical transport in ion beam created InAs nanospikes

This article has been downloaded from IOPscience. Please scroll down to see the full text article.

2012 Nanotechnology 23315301

(http://iopscience.iop.org/0957-4484/23/31/315301)

View the table of contents for this issue, or go to the journal homepage for more

Download details:

IP Address: 141.211.173.82

The article was downloaded on 26/06/2013 at 15:01

Please note that terms and conditions apply. 


\title{
Electrical transport in ion beam created InAs nanospikes
}

\author{
K A Grossklaus, J R Jokisaari, X Q Pan and J M Millunchick \\ Department of Materials Science and Engineering, University of Michigan, Ann Arbor, MI 48109-2136, \\ USA
}

E-mail: joannamm@umich.edu

Received 4 May 2012, in final form 20 June 2012

Published 13 July 2012

Online at stacks.iop.org/Nano/23/315301

\begin{abstract}
Ion beam irradiation has previously been demonstrated as a method for creating nanowire-like semiconductor nanostructures, but no previous studies have reported on the electrical properties of those structures. In this work we describe the creation and in situ transmission electron microscopy electrical characterization of nanoscale InAs spike structures on both InAs and InP substrates fabricated using a focused ion beam erosion method. Those InAs 'nanospikes' are found to possess internal structures with varying amounts of ion damaged and single crystalline material. Nanospike electrical behavior is analyzed with respect to model electronic structures and is similar to cases of barrier limited conduction in nanowires. The different electrical responses of each nanospike are found to be the result of variation in their structure, with the conductivity of InAs nanospikes formed on InAs substrates found to increase with the degree of nanospike core crystallinity. The conductivity of InAs nanospikes formed on InP substrates does not show a dependence on core crystallinity, and may be controlled by the other internal barriers to conduction inherent in that system.
\end{abstract}

(Some figures may appear in colour only in the online journal)

\section{Introduction}

The creation of semiconductor nanostructures is currently an active area of research, and methods for producing new types of nanostructures with unique properties are actively being sought. In particular, the creation of high aspect ratio or one-dimensional (1D) semiconductor nanostructures has seen significant study, as these structures have been identified as promising for use in a variety of electronic, optoelectronic, and photonic applications [1-3]. Nanostructures may also provide improvements over bulk materials in thermoelectric applications, either through quantum confinement effects due to their small size or because their structures may be designed to effectively scatter phonons leading to lower thermal conductivity [4, 5]. Specifically, high aspect ratio semiconductor nanowires have been shown to conduct electricity effectively but also possess poor thermal conductivity down their length due to their structure [6-8]. 1D nanostructures have most often been created using bottom-up growth methods such as metal particle catalyzed vapor-liquid-solid (VLS) growth and selected-area epitaxial growth using templates or masks [9]. However, ion irradiation methods may also be a viable route for the production of nanowire-like structures. Broad beam ion irradiation and erosion of III-V semiconductor surfaces has already been used to create high aspect ratio cone and pillar nanostructures using several different materials, including GaAs [10], InP [11-13], and GaSb [14, 15]. Recently focused ion beam (FIB) irradiation and erosion has also been used to create tall cone or spike structures on InAs, InAs/InP heterostructures [16], InSb, and $\mathrm{InSb} / \mathrm{GaAs}$ heterostructures [17]. As a synthesis route, ion erosion may offer greater simplicity relative to nanowire growth methods that require catalyst deposition or masking. Ion erosion produced 1D structures may also possess ion disrupted internal structures with low thermal conductivities, making them useful in thermoelectric applications. Unlike the case of traditionally grown nanowires, no reports of the transport properties of semiconductor nanostructures created by ion erosion can be found in the literature. If ion beam created nanostructures are to be used in devices and are to compete with more commonly grown nanowires in specific 
applications their transport properties and the factors that control them must be determined.

This work examines the structural and electrical characterization of nanoscale semiconductor spike structures, termed 'nanospikes', created by normal incidence $30 \mathrm{kV}$ $\mathrm{Ga}^{+}$FIB irradiation of undoped InAs/n ${ }^{+}$InAs and undoped InAs $/ n^{+}$InP film heterostructures. Structural and electronic characterization of nanospikes formed using both $\mathrm{InAs} / \mathrm{n}^{+}$ InAs and $\mathrm{InAs} / \mathrm{n}^{+} \mathrm{InP}$ heterostructures was carried out by standard transmission electron microscopy (TEM) techniques and a recently available in situ electronic probing technique in which a steerable, nanoscale tungsten probe is used within the TEM to contact the sample. This in situ technique provides correlated electronic and structural information for individual nanospikes. The nanospikes were found to have a range of inhomogeneous structures whose electronic responses varied significantly. The results of the electronic testing are discussed with respect to variation in nanospike structure and a simple electronic band structure derived considering the spike/substrate and spike/probe contacts, and finally compared to similar studies of nanowire transport in the literature.

\section{Experimental details}

Undoped InAs films for nanospike production were grown on (001)-oriented $\mathrm{n}^{+}$-doped InAs and $\mathrm{n}^{+}$-doped InP substrates using molecular beam epitaxy (MBE). The $\mathrm{n}^{+}$InAs wafers had a manufacturer specified carrier concentration of $2.0 \times$ $10^{18} \mathrm{~cm}^{-3}$ and resistivity of $3.8 \times 10^{-4} \Omega \mathrm{cm}$. The $\mathrm{n}^{+}$InP wafers had a manufacturer specified carrier concentration of $4.8 \times 10^{18} \mathrm{~cm}^{-3}$ and resistivity of $0.001 \Omega \mathrm{cm} .500 \mathrm{~nm}$-thick homoepitaxial InAs films and heteroepitaxial InAs films on InP were grown at temperatures between $460^{\circ} \mathrm{C}<T<$ $470^{\circ} \mathrm{C}$, at a rate of $0.2-0.35$ monolayers per second, and under an $\mathrm{As}_{4}$ overpressure in an EPI $930 \mathrm{MBE}$ system.

The method of nanospike creation and their formation mechanism have been previously reported in detail [16], and will only be described in brief here. Production of nanospikes directly on flat InAs film specimens was accomplished by placing the InAs or InAs/InP specimens in an FEI Nova Nanolab dual-beam FIB/scanning electron microscope (SEM) system and then FIB irradiating the InAs films at room temperature using a normal incidence, $30 \mathrm{keV} \mathrm{Ga}^{+}$ion beam. Irradiation was carried out using a beam current of $\sim 7 \mathrm{pA}$ and spot dwell times of $100 \mathrm{~ns}$. The FIB was scanned over $5 \mu \mathrm{m} \times 5 \mu \mathrm{m}$ areas repeatedly using a $50 \%$ spot overlap to provide even irradiation of the entire exposed area. SEM was used to monitor the nanospikes during their formation and characterize them afterwards. The total number of FIB passes and corresponding ion dose necessary to produce the final distribution of nanospikes varied from sample to sample, but generally fell within the range of 10000 to 15000 passes and $1.2 \times 10^{17}$ to $1.9 \times 10^{17}$ ions $\mathrm{cm}^{-2}$, respectively.

Both InAs $/ n^{+}$InAs homoepitaxial and $\mathrm{InAs} / \mathrm{n}^{+} \mathrm{InP}$ heterostructure nanospike samples were examined by TEM, and cross-sectional samples for TEM imaging and electrical testing were produced using a combination of a modified
H-bar TEM sample preparation method [18], followed by FIB irradiation to produce the nanospikes. After growth of undoped InAs films by MBE, a cleaved cross-section from each specimen was mechanically thinned to $<100 \mu \mathrm{m}$ thickness and then attached with epoxy to a flat Mo TEM ring grid. The cross-sectional sample was then placed into the Nova dual-beam FIB/SEM system and a section of the sample was further thinned using $30 \mathrm{kV}$ FIB cleaning cross-section patterns to a thickness of $<5 \mu \mathrm{m}$. All sample thinning was carried out before nanospike creation in order to limit redeposition of sputtered material in the regions containing nanospikes. Nanospikes were then created by irradiating $5 \mu \mathrm{m}$-wide regions of the thinned area. Care was taken to align the thinned cross-section for normal incidence FIB irradiation. Nanospikes created in this manner projected above the thicker region, allowing them to be viewed in transmission without the need for any additional sample preparation. In the case of InAs $/ \mathrm{n}^{+}$InP heterostructure nanospike samples, two additional FIB cuts completely through the InAs film into the underlying InP were made on both sides of the thinned region before nanospike creation. This was done to ensure that the conduction path from the nanospikes to the carbon paint and Mo grid passed through the InP substrate. After nanospike creation, a small amount of conductive colloidal carbon paint was placed on the portions of the cross-section epoxied to the Mo grid to ensure good electrical contact.

Cross-sectional nanospike TEM samples were examined using a JEOL 3011 TEM operated at $300 \mathrm{kV}$. Bright-field (BF) and high-resolution TEM (HRTEM) imaging for structural characterization of the nanospikes was carried out with the sample tilted to a [110]-type zone-axis. Following initial imaging, the nanospike TEM samples were placed in a Nanofactory Instruments ${ }^{\text {TM }}$ STM-TEM Electrical Probing double-tilt holder and inserted into a JEOL 2010F TEM operated at $200 \mathrm{kV}$ for simultaneous imaging and electrical testing. The Nanofactory Instruments ${ }^{\mathrm{TM}}$ holder contains a piezo-electric actuator designed to allow a conductive probe to be directed with nanoscale accuracy into contact with a TEM sample. Tungsten needle probes produced by electrochemical etching were used to contact the tip of each nanospike. The voltage of the probe relative to the holder could then be ramped to positive or negative values up to $\pm 10 \mathrm{~V}$, with the current through the probe to the holder recorded externally during the voltage ramp. When recording data for quantitative analysis, a maximum current range of $10 \mu \mathrm{A}$ was used, with a corresponding noise level of $\sim 10 \mathrm{nA}$ at zero bias. Using this in situ TEM nanoprobe testing method, the current-voltage $(I-V)$ response of individual nanospikes could be recorded while simultaneous TEM imaging allowed the point of contact between the $\mathrm{W}$ probe and nanospike to be unambiguously determined. Multiple sweeps through negative and positive voltages were conducted for each nanospike, with the first few voltage ramps limited to low bias, generally $\pm 1-2 \mathrm{~V}$, and correspondingly low currents. By repeating low-voltage ramps several times, it was possible to verify the repeatability of the electrical results obtained. Further voltage ramps were run to higher voltages and currents. The results of electrical testing were analyzed with 

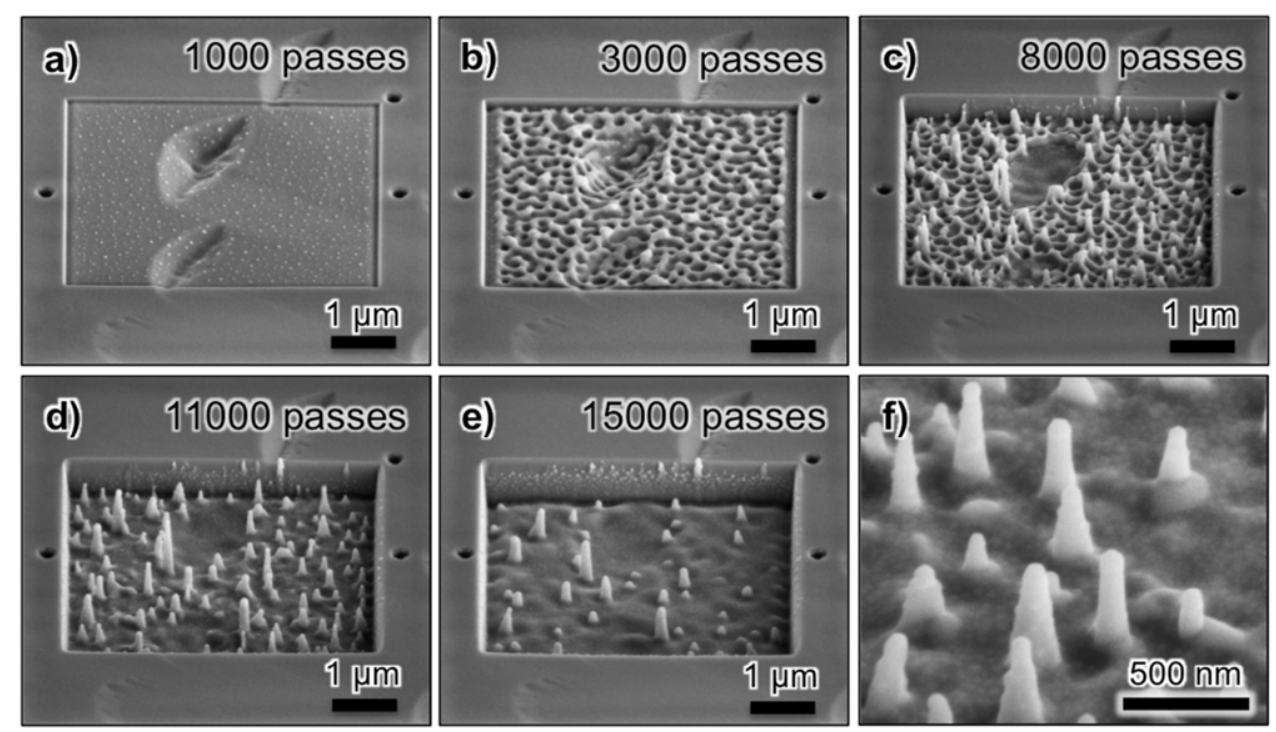

Figure 1. SEM images showing a region of rough InAs film grown on $\mathrm{n}^{+}$InP through the process of irradiation with a $7.3 \mathrm{pA}$ FIB using a $100 \mathrm{~ns}$ spot dwell time. (a)-(e) show regions of InAs/InP at various points during the nanospike creation process, with the number of beam passes delivered indicated in the upper right hand corner of each image. The three circular holes outside the irradiated region in these images were milled before irradiation for image alignment purposes. (f) shows a higher magnification image of nanospikes representative of those produced in this study using an InAs/InP heterostructure.

respect to the physical size of different nanospike structural features measured using cross-sectional TEM images taken before and during electrical testing.

\section{Results and discussion}

Nanospikes were observed to form as a result of FIB irradiation, with the location and density of nanospikes depending on the starting morphology of the InAs film and changes in surface morphology as ion erosion of the film proceeds [16]. For both the InAs $/ \mathrm{n}^{+}$InAs and InAs $/ \mathrm{n}^{+} \mathrm{InP}$ cases, the process of nanospike formation occurs in a similar manner. Figure 1 shows an $\mathrm{InAs} / \mathrm{n}^{+} \mathrm{InP}$ sample at several different points during the nanospike formation process. Initially In droplets form on the InAs film due to preferential sputtering of As during FIB irradiation [19, 20] (figure 1(a)). With continued FIB erosion, the InAs film surface transitions to an uneven web-like morphology covered with In droplets or globules (figure 1(b)). At this point some of the In droplets, which were initially mobile under the ion beam, become stationary at apexes of the uneven surface. In doing so they mask the underlying InAs, resulting in the formation of nanospikes as the surrounding material is eroded away (figures 1(c) and (d)). For the InAs $/ \mathrm{n}^{+}$InAs case, the web-like morphology persists and new nanospikes may begin to form so long as irradiation continues. In the InAs $/ \mathrm{n}^{+}$InP case, once the InAs film has been eroded away to expose the underlying InP, no new nanospikes form and existing nanospikes will begin to erode away (figure 1(e)). This is due to the inability of InP to form large In droplets or produce enough excess In atoms to maintain existing ones [20]. The inability of InP to support nanospike formation can be exploited to control the locations at which nanospikes form. Using a FIB current of $\sim 7 \mathrm{pA}$ and $100 \mathrm{~ns}$ spot dwell time, nanospikes could be created from InAs $/ \mathrm{n}^{+}$InAs samples with an average density of $2.84 \pm 0.8$ spikes $\mu \mathrm{m}^{-2}$, a range of heights with an average of $400 \pm 200 \mathrm{~nm}$, and an average diameter measured at full-width half-maximum (FWHM) height of $120 \pm 25 \mathrm{~nm}$. Using the same FIB parameters and a $500 \mathrm{~nm}$-thick InAs film, nanospikes could be created from InAs $/ \mathrm{n}^{+} \mathrm{InP}$ samples with an average density of $1.98 \pm 0.5$ spikes $\mu \mathrm{m}^{-2}$, in a range of heights with an average of $300 \pm 100 \mathrm{~nm}$, and with an average diameter measured at FWHM height of $110 \pm 30 \mathrm{~nm}$. The maximum observed nanospike height was approximately $975 \mathrm{~nm}$.

Nanospike creation on the thinned region of crosssectional TEM specimens occurs by the same process and with similar results to nanospikes produced on flat film samples. Cross-sectional bright-field TEM examination of those nanospikes revealed that the nanospikes have inhomogeneous internal structures, with both InAs $/ n^{+}$InAs and $\operatorname{InAs} / \mathrm{n}^{+} \mathrm{InP}$ nanospikes possessing the same basic structural features. All the nanospikes examined were capped with In metal tips and possessed an ion damaged outer layer that was a combination of amorphous material and many small, randomly oriented crystalline regions. Beneath the outer ion damaged layer the cores of the nanospikes had structures covering a range from almost fully crystalline to entirely disrupted by ion damage. Figure 2 contains bright-field TEM images taken of InAs $/ \mathrm{n}^{+}$InAs nanospikes, showing the range of structures. High-resolution TEM imaging of the crystalline sections of the nanospike cores confirmed that they were single crystals that matched the structure and orientation of the original InAs film from which they formed. Those single crystalline cores possessed crystalline defects at the interface with their ion damaged shell, but no extended defects such as twin boundaries crossed them. Spikes with partial or fully damaged cores also showed 

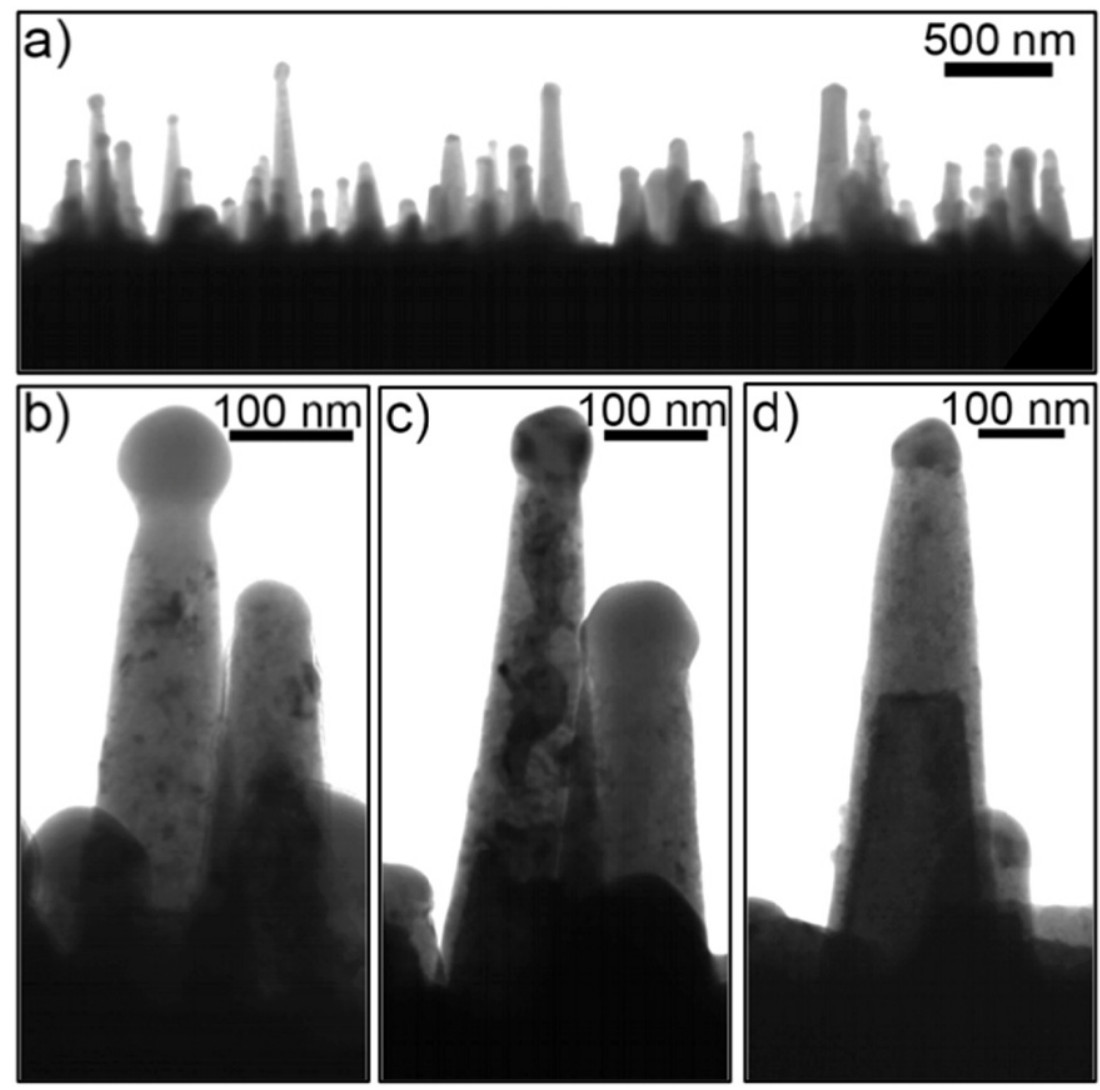

Figure 2. Bright-field TEM images of InAs/ $\mathrm{n}^{+}$InAs nanospikes. (a) shows a cross-sectional view through many spikes with different structures, (b) shows a nanospike with fully disrupted core, (c) shows a nanospike with a partially disrupted core, and (c) shows a nanospike with a single crystalline core in its lower half and ion disrupted upper half.

variation in the extent of their damage, with differing amounts of amorphous material (lighter contrast) and regions of local crystallinity (darker contrast), as seen in figure 2 .

The contact for electronic testing of the InAs $/ \mathrm{n}^{+}$InAs nanospikes in the TEM was made by driving the tungsten probe into their In droplet caps. Quantitative analysis of InAs nanospike electrical response uses the results from the first low-voltage scans with good electrical contact for each tested nanospike, which are assumed to be representative of the pristine spike. An example of an InAs $/ n^{+}$InAs nanospike electrical test is given in figure 3. Figure 3(a) shows a plot of the raw $I-V$ data from an InAs nanospike that underwent three voltage ramps. That nanospike's partially disrupted core is shown in figure 3(b) before contact with the nanoprobe. The first and second voltage ramps to -1 and $+1 \mathrm{~V}$ respectively produced smooth $I-V$ curves that were considered the electrical response of the undamaged nanospike. Figure 3(c) shows the nanospike following the first two voltage ramps. The third voltage scan consisted of a ramp to $+2 \mathrm{~V}$, during which the upper half of the nanospike decomposed and the remainder of its structure was disrupted (figure 3(d)). The point at which the nanospike began to decompose is indicated on the plot. The electrical behavior shown in figure 3 is typical of the InAs $/ n^{+}$InAs nanospike electrical tests conducted in this work. In cases where higher voltages caused damage or microstructural changes slowly enough that the process could be observed, the nanospike structure either disrupted along the spike core followed by decomposition or damage initiated directly below the In cap.

Figure 4 shows plots of the current density $(J)$ as a function of field strength $(E)$ for several InAs $/ \mathrm{n}^{+}$ InAs nanospikes. The diameter $\left(D_{\mathrm{c}}\right)$ and corresponding approximated contact area of the In droplet to the nanospike body (measurement location shown in figure 5) was chosen for determining $J$ because it was the narrowest part of the conduction path and thus likely the limiting cross-section. The electric field strength was determined by dividing the voltage by the total height of the semiconductor portion of each nanospike $\left(h_{\mathrm{s}}\right)$, found by subtracting the height of each spike's In cap from its total height. Using $D_{\mathrm{c}}$ and $h_{\mathrm{s}}$, the resistivities of the nanospikes were calculated at specific voltages for comparison. The resistivities of the InAs $/ \mathrm{n}^{+}$InAs nanospikes at -0.2 and $+0.2 \mathrm{~V}$ ranged from 0.0003 to $0.082 \Omega \mathrm{m}$. From figure 4 , it can be seen that all the nanospikes show a non-linear $J-E$ response that is either nearly symmetric or asymmetric across voltage polarity. There is also significant spread in the magnitude of the $J-E$ responses of the different spikes, with their resistivity values varying over several orders of magnitude.

The spread in the electrical response data of each tested InAs $/ n^{+}$InAs nanospike can be partially explaining by examining the effects that differences in nanospike structure are expected to have on electrical transport. As revealed by TEM characterization, there are large differences between the 

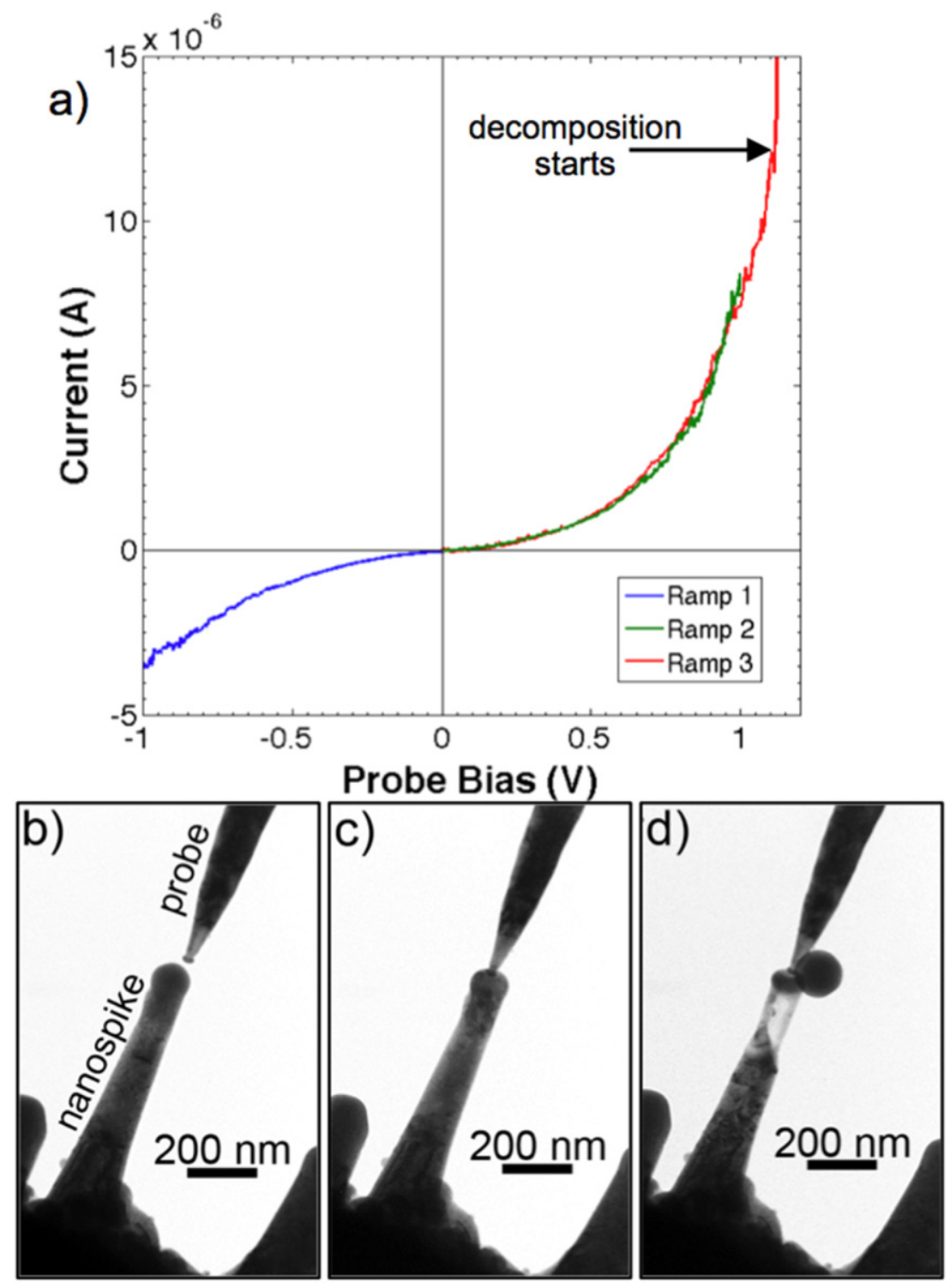

Figure 3. Full data set of $I-V$ curves and TEM images for the electrical testing of an InAs nanospike. (a) shows the raw $I-V$ data collected during testing. Image (b) shows the nanospike before the probe has made contact, (c) shows after voltage ramps 1 and 2 were completed, and (d) shows the nanospike after voltage ramp 3.

microstructures of each spike. All the nanospikes have an In droplet cap and outer ion damaged layer. However, the cores of the nanospike vary from fully disrupted amorphous and polycrystalline material to continuous single crystals (figure 2). Observations of microstructural changes to the core at high voltage suggest that carrier transport at least partially occurs through this region. The portion of each InAs nanospike core that is a continuous single crystal is readily observable through bright-field TEM imaging, and the degree to which each nanospike core is crystalline may be compared to its resistivity. An approximate single crystalline volume fraction for each nanospike was found using

$$
f_{\mathrm{c}}=\frac{h_{\mathrm{c}} w_{\mathrm{c}}^{2}}{h_{\mathrm{s}} w_{\mathrm{s}}^{2}}
$$

where $h_{\mathrm{c}}$ is the height of the crystalline core, $w_{\mathrm{c}}$ is the crystalline core width at its FWHM height and $w_{\mathrm{s}}$ is the spike FWHM. The locations of those measurements are shown schematically in figure 5. Figure 6 shows a plot of nanospike resistivity values at +0.2 and $-0.2 \mathrm{~V}$ versus single crystalline volume fraction for those electrically tested InAs $/ n^{+}$InAs nanospikes whose core structure could be determined. Because data from only seven nanospikes are represented in the plot and there is considerable scatter in the data, it is not reasonable to fit a specific resistivity versus crystalline fraction trend curve to the data in figure 6. Nonetheless, the data in the plot shows that, in general, nanospikes with the lowest resistivity also have the highest single crystalline volume fraction and higher resistivity nanospikes have a lower crystalline volume fraction. That a large single crystalline volume fraction corresponds to low nanospike resistivity is not unexpected, as single crystalline regions that still match the structure of the InAs substrate and are in good contact with it should be more conductive 


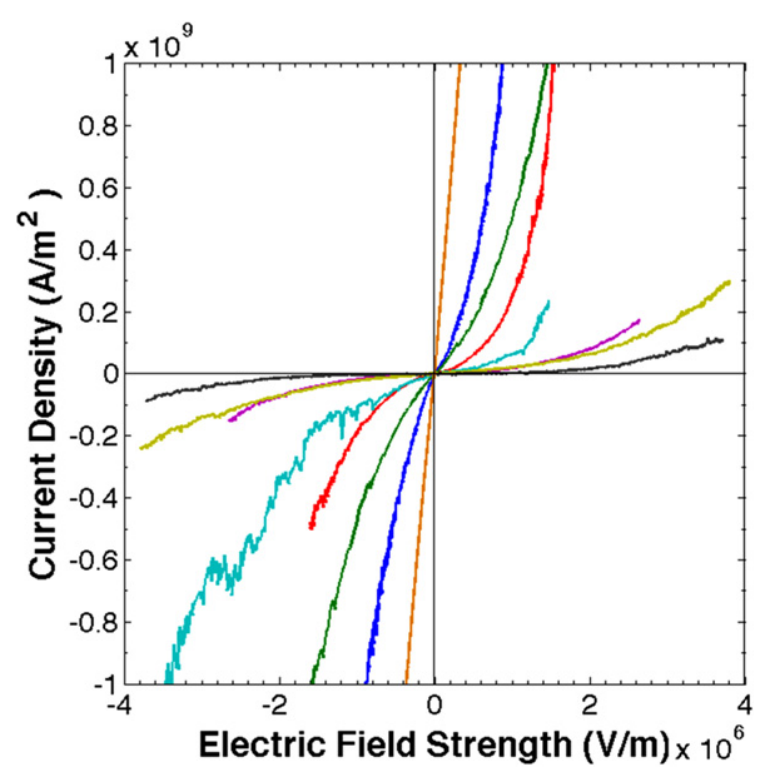

Figure 4. A plot of current density $(J)$ versus electric field strength $(E)$ from testing of eight different InAs $/ \mathrm{n}^{+}$InAs nanospikes.

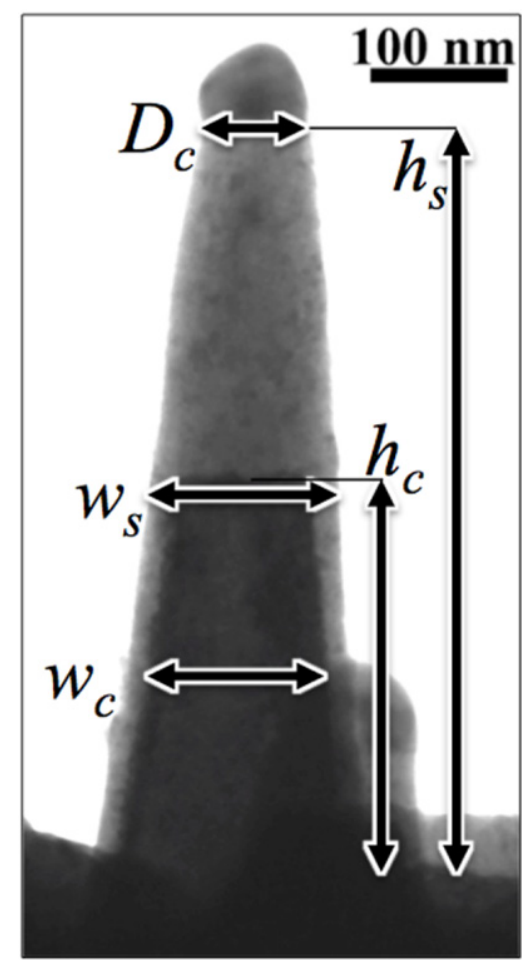

Figure 5. TEM image of an InAs/n $\mathrm{n}^{+}$InAs nanospike with a partial single crystalline core showing the location from which nanospike and core dimensions were measured for analysis.

than ion disrupted regions. Even between those nanospikes that have ion disrupted cores there is significant scatter in resistivity, which is likely due to structural differences. TEM characterization showed that the disrupted nanospike cores consisted of intermixed amorphous material and small regions of local crystallinity, and the unique structure of each disrupted nanospike core may be responsible for the additional variation in nanospike conductivity.

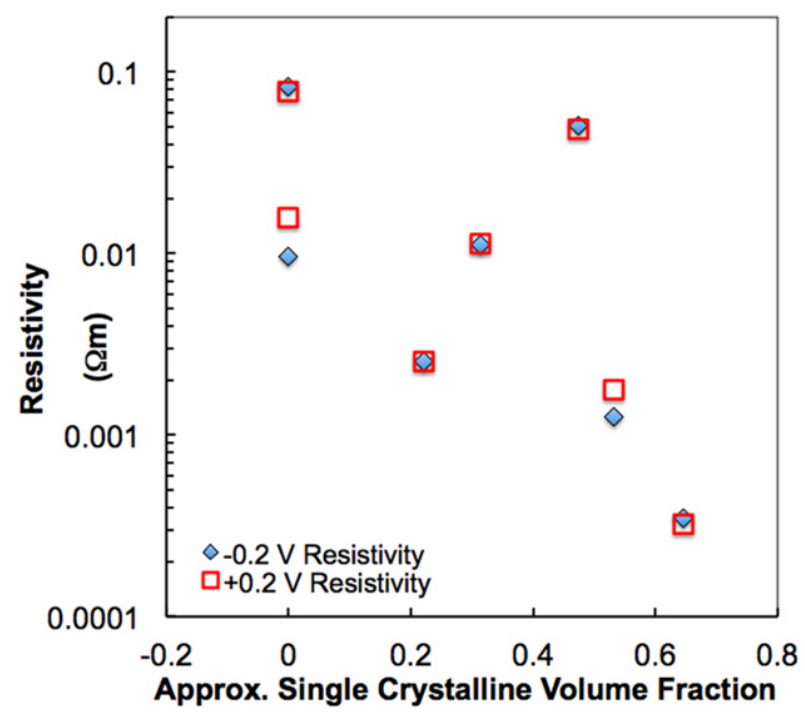

Figure 6. InAs nanospike +0.2 and $-0.2 \mathrm{~V}$ resistivity values plotted versus their approximate single crystalline core volume fraction.

Structural differences likely explain the variation in InAs $/ n^{+}$InAs nanospike resistivity, but the reason for their nonlinear $I-V$ response still needs to be determined. Individually the positive and negative polarity $J-E$ responses of the InAs nanospikes plotted in figure 4 show a smooth, nonlinear voltage dependence across the entire plotted range with no sudden changes in slope evident. This was verified visually and by plotting the instantaneous linear slope between every pair of points in each $I-V$ data set and noting the absence of any inflection points or sharp changes in those $\mathrm{d} I / \mathrm{d} V$ versus $V$ plots. It should be noted that at very low biases $(<0.1 \mathrm{~V})$ noise in the data makes analysis difficult and may have obscured a transition. Experiments where the nanoprobe was driven into unirradiated, undoped InAs film near the thinned region of the TEM sample resulted in a linear $I-V$ response, indicating that the non-Ohmic nanospike response is characteristic of the ion-induced nanospikes and not the homoepitaxially grown undoped InAs $/ n^{+}$InAs film structure used to produce the nanospikes.

Other authors have previously studied electrical transport in a variety of nanowire systems, including examinations of III-V nanowires. Of those examples in the literature, many employed a simple 2-contact or nanoprobe experimental setup and show results resembling the homoepitaxial InAs nanospike behavior seen in this work. Those studies identify a number of different mechanisms that limit conduction in different nanowires junctions and cause them to exhibit non-linear/non-Ohmic $I-V$ behavior. A series of 2-contact and SEM nanoprobe experiments have identified space-charge limited (SCL) conduction, characterized by a transition from $I \alpha V$ behavior at low bias to $I \alpha V^{2}$ behavior at higher biases and a symmetric positive-negative polarity $I-V$ response, in GaAs [21], GaN [22-24], and InAs nanowires [8, 24]. Other studies of GaAs nanowires have identified carrier trap limited behavior, characterized by a symmetric $I-V$ response and very low conduction at small bias followed by a sharp 
turn-on at higher bias [21, 25]. Finally, another series of 2-contact and TEM nanoprobe studies have identified contact barrier limited (also called injection limited) conduction as a cause of non-Ohmic $I-V$ response in nanowires [26-29]. In those studies non-Ohmic $I-V$ behavior was concluded to be the result of Schottky barriers at the two metal-nanowire junctions, with conduction controlled by thermionic emission of carriers over and tunneling through the barriers [30]. Schottky barrier limited conduction does not show a transition in voltage dependence and may produce symmetric or asymmetric $I-V$ results, depending on the difference in the contact barrier heights.

The general form of the InAs nanospike $I-V$ response is most consistent with Schottky barrier limited conduction [26-28]. For discussion of the $\mathrm{InAs} / \mathrm{n}^{+}$InAs case, a simple junction will be considered, consisting of a nanospike created from undoped InAs film, the $\mathrm{n}^{+}$InAs substrate with carrier concentration of $N_{\mathrm{D}}=2 \times 10^{18} \mathrm{~cm}^{-3}$, the In droplet at the top of the nanospike as the metal contact to the spike, and the junction between the $\mathrm{n}^{+}$InAs and the conductive carbon paint applied to the TEM sample to ensure good electrical contact to the Mo grid and TEM holder. Figure 7 shows a simplified band structure to match that junction. The relevant metal work functions, $\varphi_{m}$, and bandgaps, $E_{\mathrm{g}}$, necessary to generate that band structure were taken from [31, 32] respectively. Schottky barriers heights, $\varphi_{\mathrm{b}}$, above the Fermi level at the In and C contacts are simply the difference between the metal work function and $\chi_{\mathrm{s}}$ semiconductor electron affinity, taken as $\chi_{\mathrm{s}}=4.9 \mathrm{eV}$ for InAs [33]. Taking the carbon paint $\varphi_{m}$ as approximately 5.0 (corresponding to the value reported for bulk polycrystalline carbon) and the In $\varphi_{m}$ as $4.09 \mathrm{eV}$, the Schottky barriers are $0.1 \mathrm{eV}$ and $-0.81 \mathrm{eV}$ at the $\mathrm{C} / \mathrm{n}^{+}$InAs and InAs nanospike/In junctions respectively. During each experiment the tungsten metal probe was driven into the conductive In droplet at the top of each nanospike, so no barrier is assumed to exist at the $\mathrm{W}$ to In junction. However, if the $\mathrm{W}$ probe was not in good electrical contact with the In tip this would introduce an additional resistance into the system. For the purposes of this model, the properties of the nanospike were assumed to be those of bulk InAs, with an intrinsic carrier concentration of $N_{\mathrm{D}}=\sim 1 \times$ $10^{15} \mathrm{~cm}^{-3}$ [34]. The Fermi level, $E_{\mathrm{f}}$, of the undoped InAs nanospike and $\mathrm{n}^{+}$InAs wafer at $300 \mathrm{~K}$ were found assuming Boltzmann statistics. This electrical analysis does not take into account effects such as Fermi level pinning due to surface states or contact resistances at interfaces, and depletion region size and band bending are only approximated. However, this model provides a simple way to examine the relative sizes of the possible different barriers to conduction in a nanospike junction.

The band structure illustrated in figure 7 shows that there are relatively low barriers to electron transport for both positive and negative probe bias. If, instead of the $\mathrm{n}^{+}$ InAs/carbon paint contact, $\mathrm{a} \mathrm{n}^{+}$InAs/Mo metal contact is considered, that junction is predicted to be Ohmic. Electrons should be able to overcome these predicted barriers at low forward or reverse biases, causing a distinct change in the $I-V$ curve's dependence on voltage at that point. So while

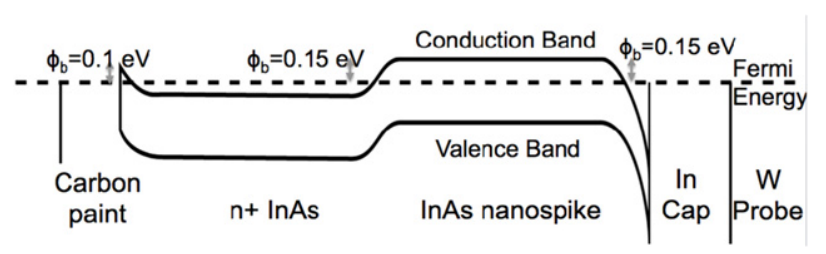

Figure 7. A simple electronic band diagram to match the proposed InAs $/ \mathrm{n}^{+}$InAs nanospike in situ TEM test junction, with the approximate energies of specific features labeled.

the conduction behavior of the InAs nanospikes appears to be barrier limited, it is not clear if the relevant barriers are due to the metal contacts or another feature of the system. All of the nanospikes tested had at least some ion damaged material between their single crystalline core and their In droplets, and it is possible that the ion damaged regions of InAs act as a barrier to electron transport and contribute to the high resistivity and nonlinear conduction behavior found for the nanospikes. The different size and structure of each nanospike's ion damaged region will correspondingly result in electronic barriers of different height and width, again providing an explanation for the spread in the conductivity of the nanospikes. The actual nature of these barriers is unclear, and thus makes fitting the experimental data with an existing theoretical model that would accurately reflect the physical features of this system difficult; regardless, a barrier resulting from ion damage likely produces the nonlinear $I-V$ behavior observed here.

Nanospikes created by FIB irradiation of InAs films grown on $\mathrm{n}^{+} \mathrm{InP}$ substrates were electrically tested in the same manner as the homoepitaxial InAs samples. The low-voltage ( 0 to $\pm 1-2 \mathrm{~V})$ portions of $\mathrm{InAs} / \mathrm{n}^{+} \mathrm{InP} I-V$ tests were used to for quantitative analysis, and testing to higher voltages and currents resulted in damage or outright destruction of the InAs $/ n^{+}$InP nanospikes. InAs $/ n^{+}$InP $I-V$ results were converted to $J-E$, and resistivity values at specific voltages were found using the same physical dimensions as the $\mathrm{InAs} / \mathrm{n}^{+}$InAs case. $J-E$ data for five $\mathrm{InAs} / \mathrm{n}^{+} \mathrm{InP}$ nanospikes are plotted in figure 8 . The resistivities of the InAs $/ \mathrm{n}^{+}$InP nanospikes at -0.2 and $+0.2 \mathrm{~V}$ range from 0.007 to $0.14 \Omega \mathrm{m}$. Again the nanospikes show a nonlinear $I-V(J-E)$ response and there is significant spread in the magnitudes of their electrical response. In general, the $\mathrm{InAs} / \mathrm{n}^{+}$InP nanospike test junctions were more resistive than the InAs $/ n^{+}$InAs nanospike junctions.

Variation in the electrical response of the InAs/InP nanospikes can be considered in terms of variation in nanospike structure in much the same way as it was for the InAs $/ \mathrm{n}^{+}$InAs nanospike case. The height and FWHM of the InAs/n $\mathrm{n}^{+}$InP nanospike crystalline cores were again used to approximate crystalline core volumes and calculate the single crystalline volume fraction using equation (1). Figure 9 shows a plot of InAs $/ \mathrm{n}^{+}$InP nanospike -0.2 and $+0.2 \mathrm{~V}$ resistivity values versus single crystalline volume fraction. System noise at low bias accounts for some of the asymmetry and scatter in the $0.2 \mathrm{~V}$ resistivity values. No trend between resistivity and single crystalline volume fraction is evident 


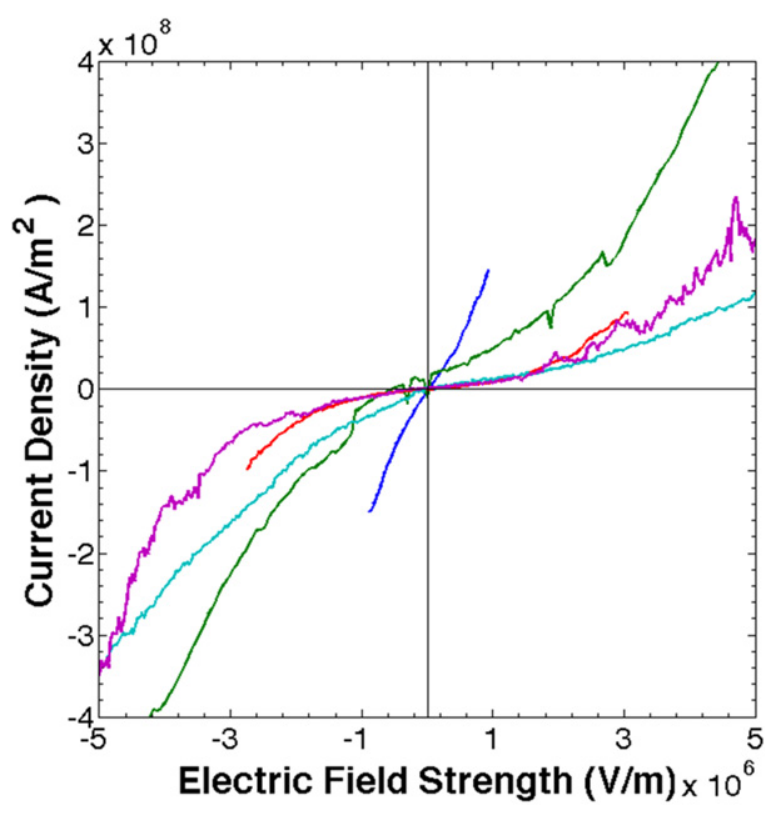

Figure 8. Plots of current density $(J)$ versus electric field strength $(E)$ from electrical testing of five $\mathrm{InAs} / \mathrm{n}^{+} \mathrm{InP}$ nanospikes.

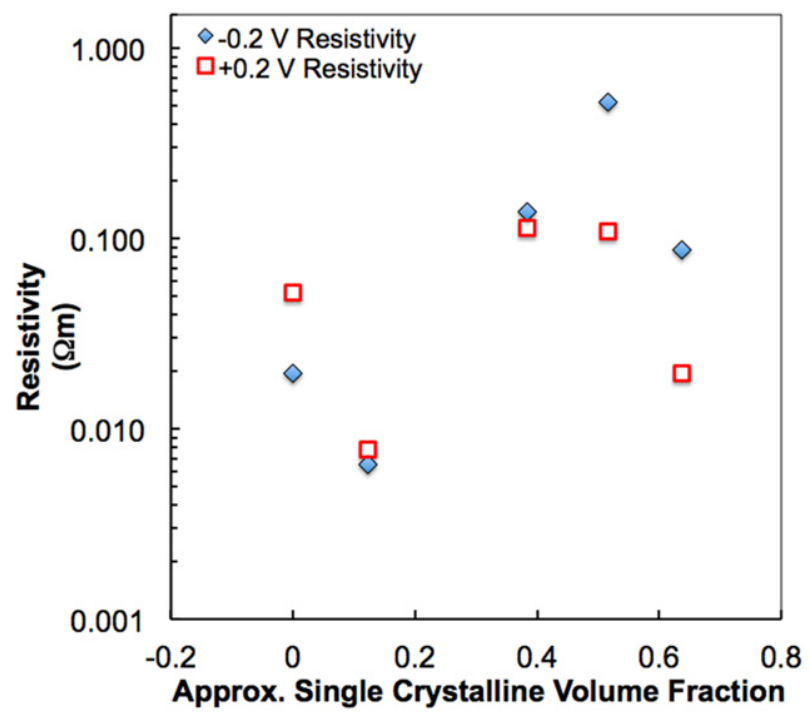

Figure 9. InAs $/ \mathrm{n}^{+} \mathrm{InP}$ nanospike +0.2 and $-0.2 \mathrm{~V}$ resistivity values plotted versus their approximate single crystalline core volume fraction.

in that scatter plot, and some of the nanospikes with higher crystalline volume fractions are also the most resistive. This does not change if the $-0.5 \mathrm{~V}$ and $+0.5 \mathrm{~V}$ resistivity values are plotted against single crystalline volume fraction. The lack of a clear trend and the scatter in figure 9 suggests that in the InAs $/ n^{+}$InP case the degree of nanospike core crystallinity is controlling conduction less than some other feature of the nanospike junction.

The general form of the InAs/n ${ }^{+}$InP $I-V$ response again resembles the cases of barrier limited conduction found in the literature. For analysis, the $\mathrm{InAs} / \mathrm{n}^{+} \mathrm{InP}$ band structure shown in figure 7 was modified to include an $\mathrm{n}^{+}$InP substrate with carrier concentration of $N_{\mathrm{D}}=4.8 \times 10^{18} \mathrm{~cm}^{-3}$ and a band

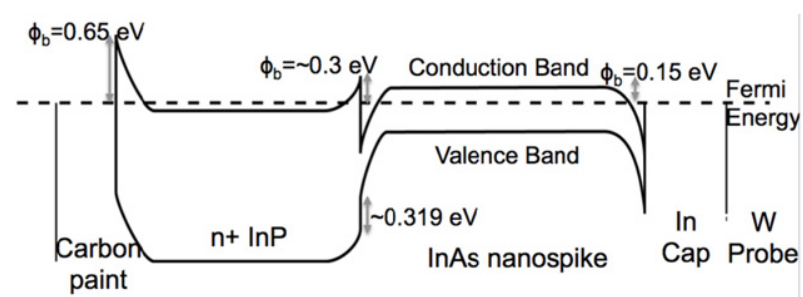

Figure 10. A simple electronic band diagram to match an InAs $/ \mathrm{n}^{+}$ InP nanospike TEM nanoprobe test junction. The calculated energies of various features are labeled on the diagram.

offset at the InAs/InP interface. Figure 10 shows a simple band structure to match that junction. The electron affinity of InP was taken as $\chi_{\mathrm{s}}=4.35 \mathrm{eV}$ [33] and the relevant $\varphi_{m}$ and $E_{\mathrm{g}}$ values were taken from the same references used for the InAs/n ${ }^{+}$InAs case. The bandgap offset at the InAs $/ \mathrm{n}^{+}$InP interface was taken as type I, with a $70 / 30$ split giving a conduction offset of $\sim 0.743 \mathrm{eV}$ and valence offset of $\sim 0.319 \mathrm{eV}$. The simple band structure developed for the $\mathrm{InAs} / \mathrm{n}^{+}$InP junction is shown in figure 10, again with no interface effects considered and depletion regions only approximated. The barrier at the $\mathrm{n}^{+} \mathrm{InP} /$ carbon contact is predicted to be $\varphi_{\mathrm{b}}=0.65 \mathrm{eV}$ and the conduction band offset barrier at the InAs/InP interface is predicted as $\varphi_{\mathrm{b}}=\sim 0.3 \mathrm{eV}$, both higher than any of the barriers predicted in the InAs $/ \mathrm{n}^{+}$ InAs nanospike electronic structure. These higher barriers may also explain why the InAs/InP nanospikes were, as a set, more resistive than the $\mathrm{InAs} / \mathrm{n}^{+}$InAs nanospikes and why the resistivities of the InAs/InP nanospike junctions appear to be less dependent on nanospike internal structure than the InAs nanospike junctions were. There may be still be a barrier due to ion damaged material in the conduction path, but if the $\mathrm{n}^{+} \mathrm{InP} / \mathrm{C}$ and InAs/n $\mathrm{n}^{+}$InP junction barriers are higher then they will control conduction in the $\mathrm{InAs} / \mathrm{n}^{+} \mathrm{InP}$ nanospike junctions. Without more complex modeling and analysis of the InAs/InP nanospike electronic structure, it is not possible to determine which barriers to electron transport play the greatest role in determining the conduction behavior of the InAs/n ${ }^{+}$InP nanospike junctions.

\section{Conclusions}

Semiconductor nanostructures created by ion erosion may possess useful or interesting electrical properties. This study is the first to examine electrical conduction in individual ion erosion created nanostructures using an in situ TEM nanoprobe approach. Nanospikes were created by FIB irradiating undoped InAs films grown on both $\mathrm{n}^{+}$InAs and $\mathrm{n}^{+}$InP substrates with $30 \mathrm{kV} \mathrm{Ga}^{+}$ions at normal incidence. Nanospikes form as In droplets created due to FIB irradiation locally act as self-assembled etch masks for the underlying InAs, locally protecting the film as the surrounding material is milled away, thus producing nanospikes. Cross-sectional TEM examination of the nanospikes showed that they have inhomogeneous structures, with ion damaged outer layers and core structures that range from single crystalline to completely 
disrupted by the ion beam. In situ probe experiments were conducted to examine the nanospike electrical response, and the nanospikes examined in this work were shown to be conductive and possess electrical responses that are influenced by their unique ion damaged structures. In the InAs $/ n^{+}$InAs case, the most conductive nanospikes were those which possessed the highest volume fraction of single crystalline InAs material, while in the $\mathrm{InAs} / \mathrm{n}^{+}$InP case no clear correlation between nanospike conductivity and core crystallinity was found. $J-E$ plots comparing the results from multiple nanospikes revealed that they show a nonlinear response resembling barrier limited conduction in semiconductor nanowires. A simple band structure model for the InAs $/ \mathrm{n}^{+}$InAs nanospike junction predicts low barriers to electron transport at the junction contacts. This may indicate that another feature, such as ion damaged material in the nanospike, may be acting as a barrier to electron transport. A similar band structure model for the InAs $/ \mathrm{n}^{+}$InP junction predicts higher barriers to conduction at the InAs/InP interface and the contact to the $\mathrm{n}^{+}$InP wafer, and these features may play a greater role in determining the conductivity of $\mathrm{InAs} / \mathrm{n}^{+}$ InP junctions.

The extent to which different features of the nanospike junctions control electrical transport in the InAs nanospikes is not completely clear and will require additional study to fully identify, but the ability of ion beam defined features to control transport may help to make ion beam created nanostructures attractive for specific device applications. In particular, the ion disrupted and inhomogeneous internal structures of the nanospikes may have low thermal conductivity, making them useful for thermoelectric applications that require materials with high electrical conductivity and low thermal conductivity for efficient operation. In general, the ability to use a focused ion beam process to produce nanoscale structures provides another method for creation of novel semiconductor nanostructures, and altering properties through control of ion damage and internal structure during ion beam processing may allow the tailoring of nanostructure transport properties to fit specific needs.

\section{References}

[1] Hu J, Odom T W and Lieber C M 1999 Chemistry and physics in one dimension: synthesis and properties of nanowires and nanotubes Acc. Chem. Res. 32 435-45

[2] Gudiksen M S, Lauhon L J, Wang J, Smith D C and Lieber C M 2002 Growth of nanowire superlattice structures for nanoscale photonics and electronics Nature $415617-20$

[3] Xia Y, Yang P, Sun Y, Wu Y, Mayers B, Gates B, Yin Y, Kim F and Yan H 2003 One-dimensional nanostructures: synthesis, characterization, and applications $A d v$. Mater. 15 353-89

[4] Dresselhaus M S, Dresselhaus G, Sun X, Zhang Z, Cronin S B and Koga T 1999 Low-dimensional thermoelectric materials Phys. Solid State 41 679-82

[5] Dresselhaus M S, Chen G, Tang M Y, Yang R G, Lee H, Wang D Z, Ren Z F, Fleurial J P and Gogna P 2007 New directions for low-dimensional thermoelectric materials Adv. Mater. 19 1043-53
[6] Li D, Wu Y, Kim P, Shi L, Yang P and Majumdar A 2003 Thermal conductivity of individual silicon nanowires Appl. Phys. Lett. 832934

[7] Zhou F, Moore A, Bolinsson J and Persson A 2011 Thermal conductivity of indium arsenide nanowires with wurtzite and zinc blende phases Phys. Rev. B 83205416

[8] Katzenmeyer A M, Leonard F, Talin A A, Toimil-Molares M E, Cederberg J G, Huang J Y and Lensch-Falk J L 2011 Observation of space-charge-limited transport in InAs nanowires IEEE Trans. Nanotechnol. 10 92-5

[9] Dick K A 2008 A review of nanowire growth promoted by alloys and non-alloying elements with emphasis on Au-assisted III-V nanowires Prog. Cryst. Growth Character. Mater. 54 138-73

[10] Tanemura M, Aoyama S, Fujimoto Y and Okuyama F 1991 Structural and compositional analyses of cones formed on ion-sputtered GaAs surfaces Nucl. Instrum. Methods B 61 451-6

[11] Nozu M, Tanemura M and Okuyama F 1994 Direct evidence for In-crystallite growth on sputter-induced InP cones Surf. Sci. 304 L468-74

[12] Chini T, Kato J and Tanemura M 1995 Monocrystalline InP surfaces normally sputtered with $\mathrm{Ar}(+)$ : SEM and TEM observations Nucl. Instrum. Methods B 95 313-8

[13] Yuba Y, Hazama S and Gamo K 2003 Nanostructure fabrication of InP by low energy ion beams Nucl. Instrum. Methods B 206 648-52

[14] Nerbø I S, Kildemo M, Roy S L, Simonsen I, Søndergård E, Holt L and Walmsley J C 2008 Characterization of nanostructured GaSb: comparison between large-area optical and local direct microscopic techniques Appl. Opt. 47 5130-9

[15] Le Roy S, Barthel E, Brun N, Lelarge A and Søndergård E 2009 Self-sustained etch masking: a general concept to initiate the formation of nanopatterns during ion erosion J. Appl. Phys. 106094308

[16] Grossklaus K A and Millunchick J M 2011 Focused ion beam creation and templating of InAs and InAs/InP nanospikes Nanotechnology 22355302

[17] Wu J H and Goldman R S 2012 Mechanisms of nanorod growth on focused-ion-beam-irradiated semiconductor surfaces: role of redeposition Appl. Phys. Lett. 100053103

[18] Szot J, Hornsey R, Ohnishi T and Minagawa S 1992 Focusedion beam micromachining for transmission electron microscopy specimen preparation of semiconductor laser diodes J. Vac. Sci. Technol. B 10575

[19] Lugstein A, Weil M, Basnar B, Tomastik C and Bertagnolli E 2004 A novel fabrication technique for crystallite growth on a (100) InAs surface utilizing focused ion beams Nucl. Instrum. Methods B 222 91-5

[20] Grossklaus K A and Millunchick J M 2011 Mechanisms of nanodot formation under focused ion beam irradiation in compound semiconductors J. Appl. Phys. 109014319

[21] Schricker A D, Forrest M D III, Wiacek R J and Korgel B A 2006 Space charge limited currents and trap concentrations in GaAs nanowires Nanotechnology 172681

[22] Talin A A, Léonard F, Swartzentruber B S, Wang X and Hersee S D 2008 Unusually strong space-charge-limited current in thin wires Phys. Rev. Lett. 10176802

[23] Talin A A, Swartzentruber B S, Léonard F, Wang X and HerseeS D 2009 Electrical transport in GaN nanowires grown by selective epitaxy J. Vac. Sci. Technol. B 27 2040-3

[24] Talin A A, Léonard F, Katzenmeyer A M, Swartzentruber B S, Picraux S T, Toimil-Molares M E, Cederberg J G, Wang X, Hersee S D and Rishinaramangalum A 2010 Transport characterization in nanowires using an electrical nanoprobe Semicond. Sci. Technol. 25024015 
[25] Katzenmeyer A M, Léonard F, Talin A A, Wong P S and Huffaker D L 2010 Poole-Frenkel effect and phonon-assisted tunneling in GaAs nanowires Nano Lett. $103067-72$

[26] Zhang Z Y, Jin C H, Liang X L, Chen Q and Peng L M 2006 Current-voltage characteristics and parameter retrieval of semiconducting nanowires Appl. Phys. Lett. 88073102

[27] Zhang Z, Yao K, Liu Y, Jin C, Liang X, Chen Q and Peng L M 2007 Quantitative analysis of current-voltage characteristics of semiconducting nanowires: decoupling of contact effects Adv. Funct. Mater. 17 2478-89

[28] Liu Y, Zhang Z Y, Hu Y F, Jin C H and Peng L M 2008 Quantitative fitting of nonlinear current-voltage curves and parameter retrieval of semiconducting nanowire, nanotube and nanoribbon devices J. Nanosci. Nanotechnol. 8 252-8
[29] Liu B, Bando Y, Wang M and Golberg D 2010 Synthesis and in situ TEM transport measurements of individual gan nanowires and nanotubes J. Nanosci. Nanotechnol. $103945-51$

[30] Sze S M, Coleman D J and Loya A 1971 Current transport in metal-semiconductor-metal (Msm) structures Solid-State Electron. 141209

[31] Haynes W M (ed) 2011 CRC Handbook of Chemistry and Physics (Cleveland: CRC Press) chapter 12

[32] Vurgaftman I, Meyer J R and Ram-Mohan L R 2001 Band parameters for III-V compound semiconductors and their alloys J. Appl. Phys. 895815

[33] Bhattacharya P 1997 Semiconductor Optoelectronic Devices (Upper Saddle River, NJ: Prentice-Hall)

[34] Oda O 2007 Compound Semiconductor Bulk Materials and Characterization (Singapore: World Scientific) 